\title{
Proton driver scenarios at CERN and Rutherford Appleton Laboratory
}

\author{
J. W. G. Thomason \\ STFC/ISIS, Didcot, OX11 0QX, United Kingdom \\ R. Garoby and S. Gilardoni \\ CERN, Geneva, Switzerland \\ L. J. Jenner \\ Imperial College London, SW7 2BW, United Kingdom, and Fermilab, \\ Batavia, Illinois 60510-5011, USA \\ J. Pasternak \\ Imperial College London, SW7 2BW, United Kingdom, and STFC/ISIS, Didcot, \\ OX11 0QX, United Kingdom \\ (Received 19 October 2012; published 6 May 2013)
}

\begin{abstract}
The concept of sharing a high-power proton accelerator (proton driver) between neutrino production and other facilities such as a high-energy collider or a spallation neutron source is an attractive, costeffective solution which is being studied in site-specific cases as part of accelerator upgrade plans at CERN and at the ISIS facility at the Rutherford Appleton Laboratory. Descriptions of these proton-driver scenarios will be given primarily in the context of the Neutrino Factory, but other neutrino production plans at CERN will also be noted.
\end{abstract}

DOI: 10.1103/PhysRevSTAB.16.054801

PACS numbers: 29.20.D-

\section{INTRODUCTION}

The proton driver at the Neutrino Factory (NF) [1] is required to deliver a proton beam of $4 \mathrm{MW}$ at a repetition rate of $50 \mathrm{~Hz}$ to the pion-production target. The proton-beam energy must be in the multi-GeV range in order to maximize the pion yield. In addition, the NF specifies a particular time structure consisting of three very short bunches separated by about $120 \mu \mathrm{s}$. To allow the muon beam to be captured efficiently, short, 1-3 ns rms, bunches are required. Each bunch from the proton driver will become a separate muon bunch train. The bunch separation is constrained by beam loading in the downstream muon accelerator systems and by the time scale for disruption of the mercury-jet target. The proton-beam parameters necessary to produce the desired number of muons in the storage rings of the NF are listed in Table I. In order to achieve such short bunches, a dedicated bunch compression system must be designed to deal with the very strong space-charge forces.

Several proton-driver schemes fulfilling these requirements have been proposed. Typically they consist of an $\mathrm{H}^{-}$-ion source followed by a radio-frequency quadru-

Published by the American Physical Society under the terms of the Creative Commons Attribution 3.0 License. Further distribution of this work must maintain attribution to the author(s) and the published article's title, journal citation, and DOI. pole, a chopper, and a linear accelerator. In the solution adopted at CERN, the final energy of the proton driver is delivered by the linac and the beam time structure must be obtained with the help of charge-exchange injection into an accumulator ring followed by fast phase rotation in a dedicated compressor ring. An alternative approach, as adopted in the Rutherford Appleton Laboratory (RAL) solution, is to use a chain of circular accelerators, typically rapid cycling synchrotrons (RCSs), where bunch compression is accomplished adiabatically in the final RCS.

TABLE I. Proton-driver requirements. A proton kinetic energy in the range 5 to $15 \mathrm{GeV}$ has been shown to provide adequate performance. The number of protons, beam radius, $\beta^{*}$, and geometric emittance correspond to the values for an $8 \mathrm{GeV}$ proton beam.

\begin{tabular}{lc}
\hline \hline Parameter & Value \\
\hline Kinetic energy & $5-15 \mathrm{GeV}$ \\
Average beam power & $4 \mathrm{MW}$ \\
& $\left(3.125 \times 10^{15} \mathrm{protons} / \mathrm{s}\right)$ \\
Repetition rate & $50 \mathrm{~Hz}$ \\
Bunches per train & 3 \\
Total time for bunches & $240 \mu \mathrm{s}$ \\
Bunch length (rms) & $1-3 \mathrm{~ns}$ \\
Beam radius & $1.2 \mathrm{~mm}(\mathrm{rms})$ \\
Rms geometric emittance & $<5 \mu \mathrm{m}$ \\
$\beta^{*}$ at target & $\geq 30 \mathrm{~cm}$ \\
\hline \hline
\end{tabular}




\section{PLANS AT CERN}

\section{A. The neutrino factory}

The CERN NF scenario would be based on the proposed $5 \mathrm{GeV}$, high-power version of the Superconducting Proton Linac (SPL) [2], which can deliver $10^{14}$ protons at the repetition rate of $50 \mathrm{~Hz}$ [3]. In the recent past, the SPL study evolved into an international collaboration whose aim is the optimization of the architecture of a pulsed superconducting high-power proton linac. The most recent design of the SPL and the description of the goals of the collaboration can be found in [4].

In the CERN scenario, the chopped beam from the SPL would be injected into an isochronous accumulator ring in which $120 \mathrm{~ns}$ long bunches are formed without the need for an rf system. The absence of synchrotron motion in the accumulator ring makes it important to study the stability of the beam in the presence of space charge. As presented in [5], transverse stability can be obtained with a suitable choice of chromaticity as shown in Fig. 1 (left panel) and longitudinal stability can be achieved by limiting the longitudinal broadband impedance to a few ohms as shown in Fig. 1 (right panel) [5]. Two-dimensional phase-space painting is used in the stripping injection into the accumulator ring, allowing the temperature of the stripping foil to be kept below $2000 \mathrm{~K}$. The beam parameters after accumulation are obtained as a compromise between the competing requirements of minimizing the heating of the injection foil, maximizing the aperture, and adequate compensation of the space-charge forces and are set to allow for rf phase rotation in the downstream compressor ring. The size of the two rings is determined by the requirement that successive bunches must arrive at the correct location in the compressor ring. The compressor ring has a large phase slip factor, which is needed for the fast phase rotation. Tracking simulations in the compressor ring have been performed using the ORBIT code [6]. The good performance of the compressor ring is demonstrated in Fig. 2 (left panel). The simulations have also been used to investigate the transverse phase space. Figure 2 (right panel) shows that the transverse space charge can be tolerated due to the limited number of turns of the beam in the compressor ring and the relatively large dispersion, which effectively lowers the tune shift by enlarging the beam size. The parameters of the accumulator and compressor rings are listed in Table II. More details of the CERN proton-driver scenario can be found in [7]. The low energy normalconducting part of the SPL is currently under construction and should become operational in the following few years as part of the LHC injector chain. The existing proton
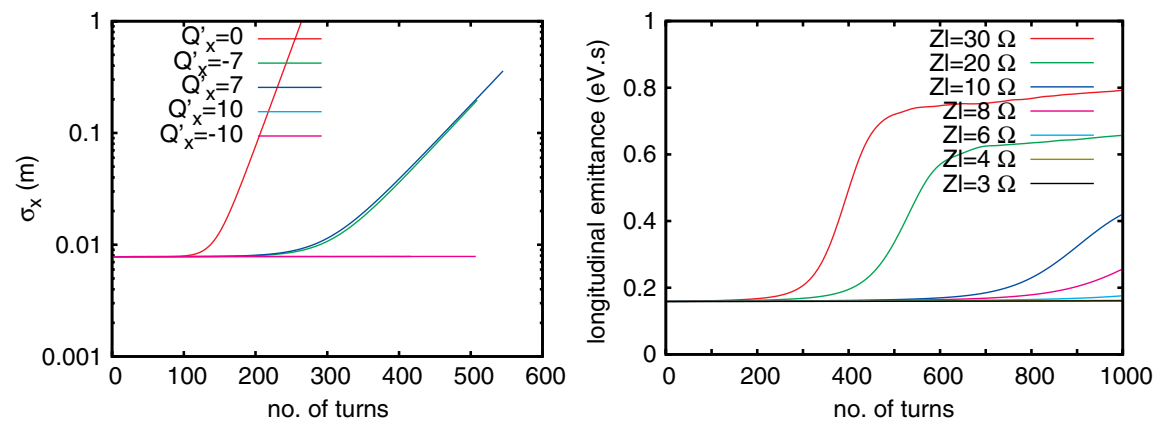

FIG. 1. Horizontal beam size evolution for various values of chromaticity assuming a transverse impedance of $1 \mathrm{M} \Omega / \mathrm{m}, Q_{R}=1$, $f_{R}=1 \mathrm{GHz}$ (left). Longitudinal emittance evolution for different values of shunt impedance (right). The lines for 3 and $4 \Omega$ lie on top of each other in the figure.
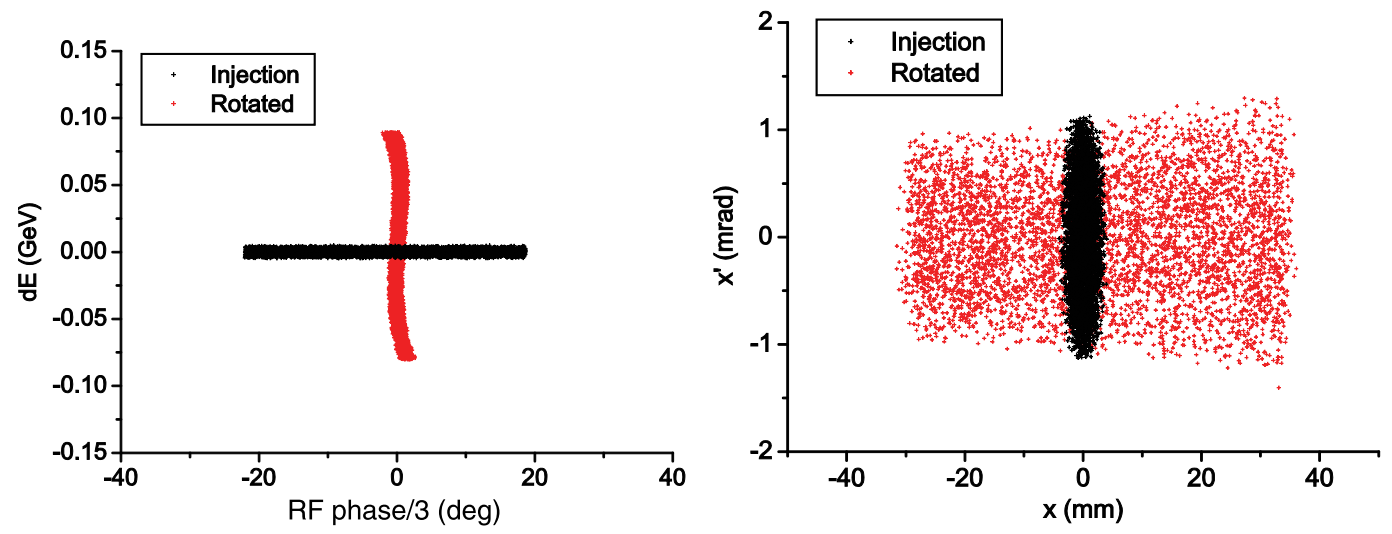

FIG. 2. Phase-space plots before and after bunch rotation. 
TABLE II. Parameters of the accumulator and compressor rings for the CERN proton-driver scenario.

\begin{tabular}{lc}
\hline \hline Parameter & Value \\
\hline Accumulator ring & \\
Circumference & $185 \mathrm{~m}$ \\
No. of turns for accumulation & 640 \\
Working point (H/V) & $7.37 / 5.77$ \\
Total bunch length & $120 \mathrm{~ns}$ \\
rms momentum spread & $0.863 \times 10^{-3}$ \\
Compressor ring & \\
Circumference & $200 \mathrm{~m}$ \\
No. of turns for compression & 86 \\
rf voltage & $1.7 \mathrm{MV}$ \\
Gamma transition & 2.83 \\
Working point & $4.21 / 2.74$ \\
\hline \hline
\end{tabular}

linac, Linac2, will be replaced soon by the more modern Linac4 [8] that will accelerate $\mathrm{H}^{-}$up to $160 \mathrm{MeV}$, before injection into the Proton Synchrotron Booster. The linac performances will match the requirements of the program of increasing the LHC luminosity.

Figure 3 shows a preliminary layout of the NF on the CERN site, using the SPL followed by a transfer channel towards the accumulator and compressor rings. This geometry is constrained by the location of Linac4 and the space needed for the muon front-end and muon acceleration chain.

\section{B. Super beams and beta beams}

As well as provision for the NF, other new proposals are being made for experiments at CERN requiring higher beam power to produce neutrinos by either exploiting existing machines or assuming the availability of beam from the $5 \mathrm{GeV}$, high-power version of the SPL.

\section{Neutrino experiments with existing accelerators}

The existing CERN accelerators deal with multiple types of particles and supply beams to numerous experiments (see layout in Fig. 4). The CNGS experiment [9] is currently operating using a $500 \mathrm{~kW}$ proton beam from the SPS and sending neutrinos to the Gran Sasso underground laboratory $730 \mathrm{~km}$ away. The SPS performance is expected to improve by the end of the decade, as a result of the on-going upgrade program of the LHC injectors [10]. The recently started LAGUNA-LBNO Design Study [11] is aimed at making use of the increased SPS beam power, tentatively set at $750 \mathrm{~kW}$, for generating a conventional $\nu_{\mu}$ beam and sending it to a

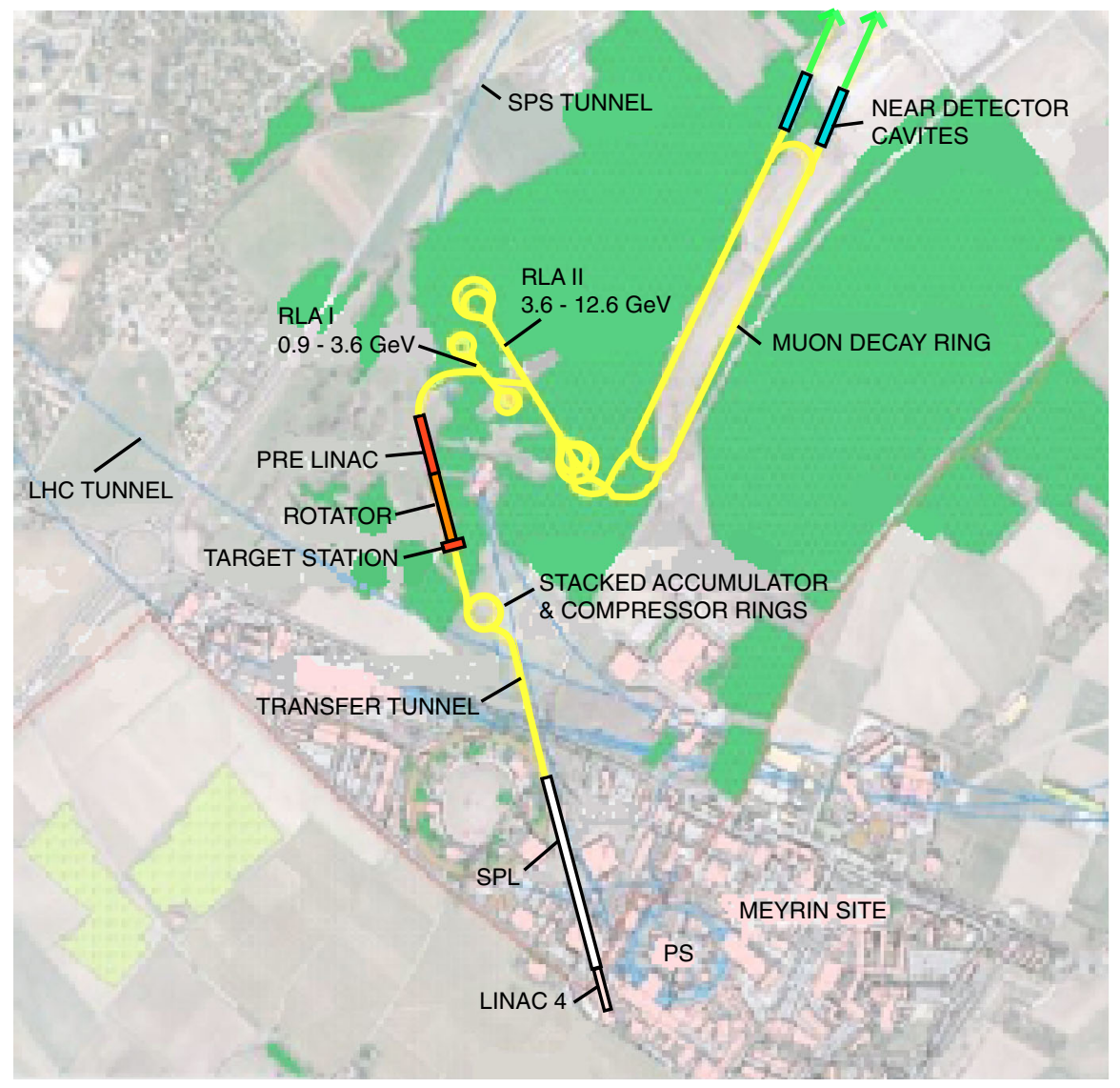

FIG. 3. Preliminary layout of the NF on the CERN site, with neutrino beams pointing at the Pyhäsalmi mine in Finland. 


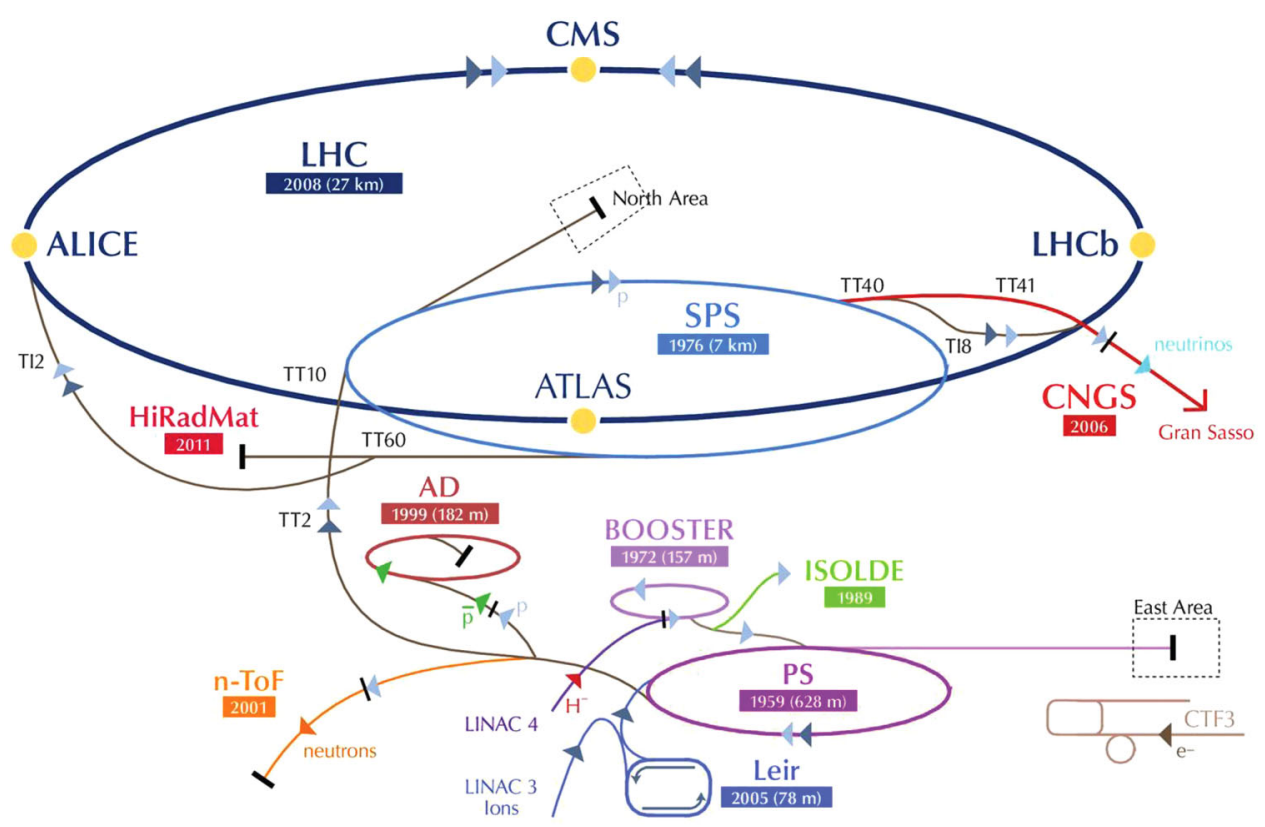

FIG. 4. Existing CERN accelerator complex with Large Hadron Collider (LHC), Super Proton Synchrotron (SPS), Proton Synchrotron (PS), Antiproton Decelerator (AD), Low Energy Ion Ring (LEIR), Linear Accelerators (LINAC), CLIC Test Facility (CTF3), CERN $\nu$ to Gran Sasso (CNGS), Isotopes Separation on Line (ISOLDE), and neutrons Time of Flight (n-ToF).

new underground experiment located in Pyhäsalmi (Finland), at a distance of $2300 \mathrm{~km}$. Beyond the upgrade of the SPS and PS complex which is foreseen in the context of the high-luminosity upgrade of the LHC, this proposal only assumes the construction of a new transfer line from the SPS to a new target area and decay tunnel oriented towards Finland.

\section{Neutrino experiments based on the SPL}

In the context of the LAGUNA-LBNO Design Study, a high-power proton synchrotron (HP-PS) will be studied which would deliver $2 \mathrm{MW}$ of beam power onto the target and decay tunnel first used by the SPS and aimed at Pyhäsalmi. The exact energy will be defined in interaction with the experimenters within LAGUNA-LBNO, but is expected to be in the range 30-50 GeV. Thereafter the accelerator will be designed using the work done for PS2 [12,13]. The injector will be a slower cycling and hence lower power version of the SPL. Unlike PS2, the HP-PS will be dedicated to neutrino production and will not be connected to the SPS.

Another possibility is that the SPL beam is accumulated in a 200-300 m circumference fixed-energy ring (which does not need to be isochronous as in the NF case), using charge-exchange injection. To generate a conventional low energy $\nu_{\mu}$ beam from $\pi$ decay, the beam is fast ejected from the accumulator onto the target. In such a scenario, a fraction of the linac beam $(\approx 200 \mathrm{~kW})$ could be diverted to a radioactive ion production system of ISOL-type to generate a beta beam [14].

\section{PLANS AT RAL}

\section{A. ISIS megawatt upgrades}

RAL is the home of ISIS, the world's most productive spallation neutron source. ISIS has two neutron producing target stations (TS-1 and TS-2), driven at 40 and $10 \mathrm{~Hz}$, respectively, by a $50 \mathrm{~Hz}, 800 \mathrm{MeV}$ proton beam from an RCS, which is fed by a $70 \mathrm{MeV} \mathrm{H}^{-}$drift tube linac [15]. Potential upgrades of the ISIS accelerators to provide beam powers of 2-5 MW in the few $\mathrm{GeV}$ energy range could be envisaged as the starting point for a proton driver shared between a short pulse spallation neutron source and the NF. Although the requirements for the NF baseline proton energy and time structure are different from those for a spallation neutron source, an additional RCS or fixed field alternating gradient (FFAG) booster bridging the gap in proton energy and performing appropriate bunch compression seems feasible.

The first stage of the upgrade path is to replace parts or all of the ISIS $70 \mathrm{MeV} \mathrm{H}^{-}$injector in order to address obsolescence issues with the present linac, and ensure reliable operation for the foreseeable future. The more exciting, but more challenging, option is to install a higher energy linac (up to $180 \mathrm{MeV}$ ) with a new optimized injection system into the present ring. This could give a substantial increase in beam power (up to 0.5 MW) [16].

The next stage is a new RCS at about $3.2 \mathrm{GeV}$ that can be employed to increase the energy of the existing ISIS beam to provide powers of around $1 \mathrm{MW}$. This new RCS would require a new building, along with a new MW-class target station. There are a number of possible candidates for this 


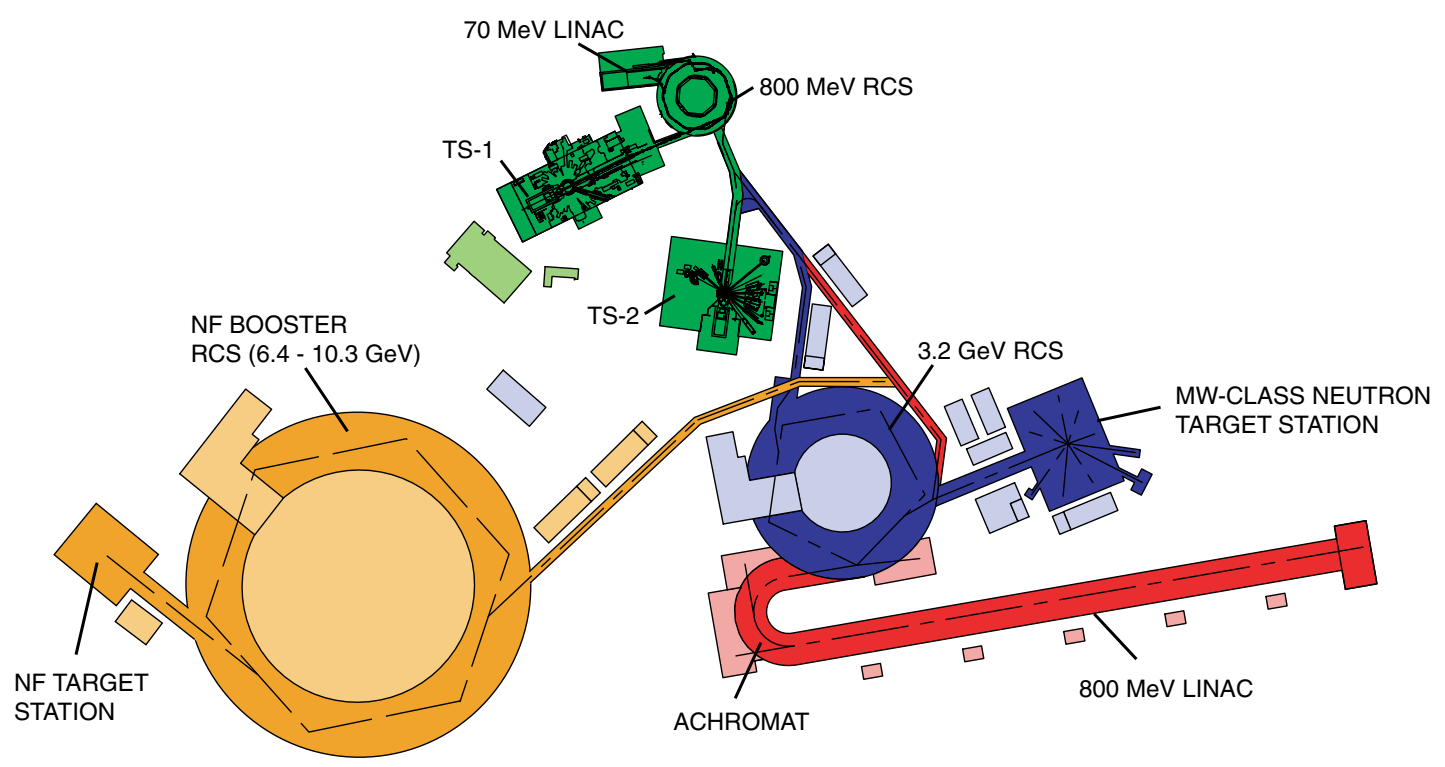

FIG. 5. Conceptual layout of the NF proton driver with ISIS (green), $\approx 3.2 \mathrm{GeV}$ RCS (blue), $800 \mathrm{MeV}$ linac (red), and dedicated NF booster (orange) within the RAL site boundary.

$50 \mathrm{~Hz}$ RCS, but studies are presently focused on a $3.2 \mathrm{GeV}$ doublet-triplet design with five superperiods (5SP) and a $3.2 \mathrm{GeV}$ triplet design with four superperiods (4SP). Both these designs will include features required for fast injection directly from the existing ISIS RCS, together with the option for optimized multiturn injection from a new $800 \mathrm{MeV} \mathrm{H}^{-}$linac [17].

The final upgrade stage is to accumulate and accelerate beam in the new RCS from a new $800 \mathrm{MeV}$ linac for 2-5 MW beams [18]. It should be noted that a significant collimation section or "achromat" would be required after the linac to provide a suitably stable beam for injection into the RCS. These upgrades to the ISIS facility are shown in Fig. 5.

\section{B. Common proton driver}

In a common proton driver for a neutron source and the NF, based on a 2-5 MW ISIS upgrade bunches of protons are shared between the two facilities at about $3.2 \mathrm{GeV}$, and a dedicated RCS or FFAG booster must then accelerate the NF bunches to meet the requirements for the NF baseline. Possible bunch sharing scenarios [19] suggest that 6.4-10.3 GeV RCS and FFAG booster designs are to be considered. Figure 5 shows the conceptual layout of the common proton driver.

Booster RCS designs [20] have concentrated on achieving the necessary acceleration and bunch compression with present-day, cost-effective RCS technology. The preliminary lattice design has been produced, but a great deal of work remains to be done to produce a full conceptual scenario. No consideration has yet been given to beam transport to the pion-production target.
FFAG options are yet to be explored, and would be based on technology which remains to be fully tested. In principle, these would offer the advantage of allowing all the bunches to be extracted to the NF target with the same energy (unlike the RCS where the $160 \mu$ s sequential extraction delay required by the NF baseline would give time for the main magnet field to vary between bunches).

Optimized longitudinal muon capture in the muon front end of the NF requires compression of the proton bunch length from the $\approx 100 \mathrm{~ns}$ for the neutron source to 1-3 ns at the NF target. Several methods have been proposed in order to reach this goal, based on either adiabatic compression during acceleration or fast phase rotation at the end of acceleration (or in an additional compressor ring).

The site-specific design at RAL is in a preliminary stage. Extensive effort on beam dynamics and accelerator engineering (and strategic research and development in a number of key areas such as high-power front ends, rf systems, stripping foils, kickers, and diagnostics) will be required before it can be regarded as viable. The common proton driver could fit onto the RAL site, on land already set aside for large facilities and research expansion, but the complete NF would require the use of part of the Harwell Oxford Campus. A possible schematic layout of the NF on the Harwell Oxford site is shown in Fig. 6. It is hoped that a new (up to $180 \mathrm{MeV}$ ) linac will be installed on ISIS between 2014 and 2020. Further upgrade stages are unlikely to be realized in the foreseeable future unless a decision is made to site the $\mathrm{NF}$ at RAL, and funding for the common proton driver is forthcoming. 


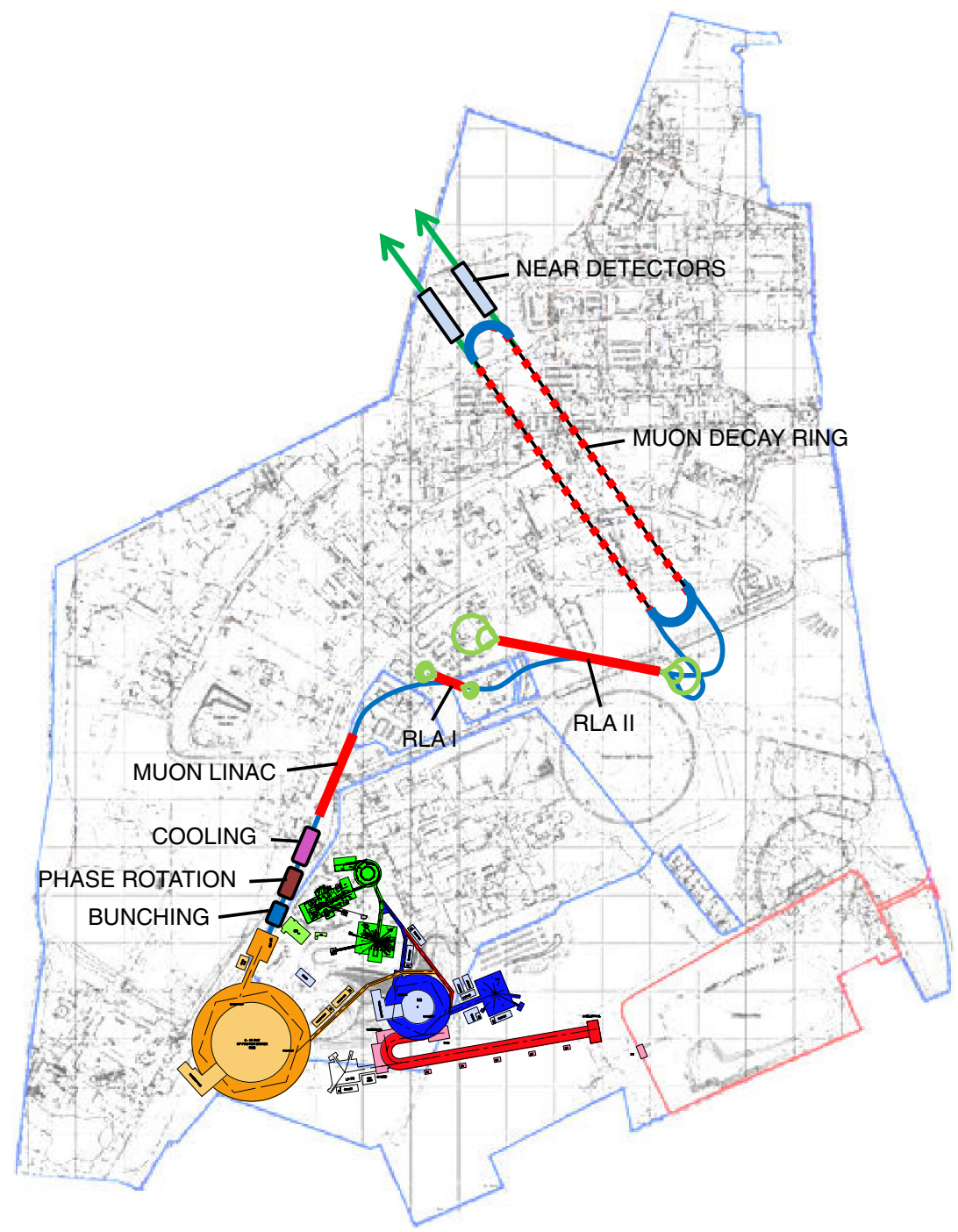

FIG. 6. Schematic layout of the NF on the Harwell Oxford site with neutrino beams pointing at Norsaq in Greenland. The proton driver is as shown in Fig. 5 and the Harwell Oxford site boundary is shown in bright blue.

\section{SUMMARY}

It seems feasible that a proton driver which could be shared between neutrino production and other facilities could be constructed at either CERN or RAL, based on upgrades already envisaged at each site. The CERN plans are considerably more mature than those at RAL, with elements of the SPL hardware having already undergone extensive testing, and with designs for dedicated NF accumulator and compressor rings well advanced. The RAL scenario is much more conceptual at this stage, and will require refinement as more detailed designs for ISIS upgrades are produced over the next few years.

\section{ACKNOWLEDGMENTS}

We acknowledge the financial support of the European Community under the European Commission Framework Programme 7 Design Study: EUROnu, Project No. 212372.
We also thank colleagues from the International Design Study (IDS-NF) collaboration for fruitful discussions concerning this work.

[1] J. Pozimski et al., in Proceedings of EUROnu12 (2012).

[2] F. Gerigk et al., Technical Report No. CERN-CERN, 2006.

[3] R. Garoby, in Proceedings of the 14th International Conference on RF Superconductivity (Helmholtz Centre, Berlin, 2009).

[4] F. Gerigk et al., Technical Report No. CERNATS-CERN, 2010.

[5] E. Benedetto, in Proceedings of the 11th International Workshop on Neutrino Factories, Superbeams and Beta Beams (2009).

[6] J. A. Holmes et al., in Proceedings of the 8th European Particle Accelerator Conference, Paris, France (EPS-AG, France, 2002). 
[7] R. Garoby et al., Technical Report No. CERNNEUTRINOFACTORY-NOTE-157, 2009.

[8] L. Arnaudon et al., CERN, Technical Reports No. CERNAB-ABP/RF and No. CARE-Note-2006-022-HIPPI, 2006.

[9] K. Elsener, Technical Report No. CERN-98-02, 1998.

[10] R. Garoby et al., in Proceedings of the 2nd International Particle Accelerator Conference, San Sebastián, Spain (EPS-AG, Spain, 2011).

[11] LAGUNA-LBNO Design Study European Commission 7th Framework Program, Grant Agreement No. 284518 (2011) [http://pprc.qmul.ac.uk/sites/default/files/DOW\% 20LAGUNA-LBNO\%20-284518-\%202011-06-15.pdf].

[12] M. Benedikt and B. Goddard, in Proceedings of the 23rd Particle Accelerator Conference, Vancouver, Canada, 2009 (IEEE, Piscataway, NJ, 2009).

[13] R. Garoby, Technical Report No. CERN-0034, 2010.

[14] "Final report on the EURISOL design study," European Commission Contract No: 515768 RIDS (2009).
[15] D. J.S. Findlay, in Proceedings of the 2007 Particle Accelerator Conference, Albuquerque, New Mexico (IEEE, New York, 2007).

[16] C.M. Warsop et al., in Proceedings of the 2nd International Particle Accelerator Conference, San Sebastián, Spain (Ref. [10]).

[17] J.W.G. Thomason et al., in Proceedings of HB2008 (JACoW, Nashville, 2008).

[18] D.C. Plostinar et al., in Proceedings of the 2nd International Particle Accelerator Conference, San Sebastián, Spain (Ref. [10]).

[19] J. W. G. Thomason and J. Pasternak, in Proceedings of the 2nd International Particle Accelerator Conference, San Sebastián, Spain (Ref. [10]).

[20] J. Pasternak and L. Jenner, in Proceedings of the 2nd International Particle Accelerator Conference, San Sebastián, Spain (Ref. [10]). 